opposite directions (in the centre of mass frame of the source). Using a more massive source, not totally annihilated by the pair production, would indeed allow better localization of the source itself, but at the cost of introducing the complication of source recoil. The more massive the source, the less the effective constraint on the initial' combined momentum of the particle pair (with respect to the centre of mass of the source). In the limit of a very massive source (say, the Earth), the directions in which the two particles were emitted would effectively be uncorrelated, and no conclusions at all could be drawn about the position of one from the detection of the other.

The net effect of the recoil for a massive source is that it really is $\Delta p_{y}$ of the source (as we used), not $\Delta v_{y}$, which is relevant in the end. One can get the same result without giving any consideration to the source at all, by realizing that the centre-of-mass position and momentum of the particle pair (in the laboratory frame) must themselves obey an uncertainty relation immediately after emission.

The geometrical contribution to the uncertainty may indeed be made small by taking $\Delta y$ of the source to be small and $d$ sufficiently long: this is implicit in our equation (5). In this case $\Delta v_{v}$ for the righthand particle will also be small, as Popper says, but what is important is $\Delta v_{y}$ for the left-hand particle. This includes a contribution from $\Delta p$, of the source, which does not depend on $d$, and it is large if $\Delta y$ of the source is small.

As regards the choice of scaling, our equation (2) is a unit of length: naturally its square is a measure of area. With this rescaling of the variables, equation (3) does follow directly from equation (1).

M.J. Collett

R. LOUDON

Department of Physics,

University of Essex,

Colchester CO4 $3 S Q, U K$

\section{A function for tubulin tyrosination?}

SIR-In a recent News and Views article!, Roy Burns reviewed some of the literature on tubulin tyrosination and proposed some models for the function of this unique post-translational modification in vivo. Unfortunately, Burns omitted to discuss some recent work that is relevant to the ideas he proposed.

The main tenet of Burns' article is whether the tyrosination state of tubulin protomers in vivo can regulate their ability to assemble into microtubules. Burns suggested that the detyrosinated (Glu) tubulin protomers in cells are compromised in their ability to polymerize into microtubules, whereas the tyrosinated (Tyr) subunits are polymerization-

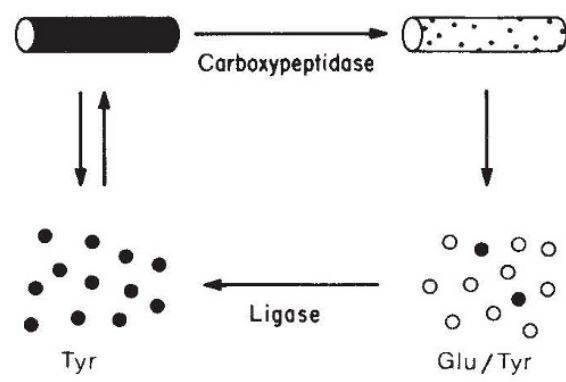

Generation of distinct populations of microtubules by the cyclic tryosination-detyrosination of $\alpha$-tubulin.

competent. But in a recent study $^{3}$, the polymerization-competence of Glu protomers in vivo was demonstrated directly by microinjection of Glu tubulin into cultured cells. Yet it is clear that, aside from this microinjection experiment, Glu protomers do not polymerize to form microtubules in vivo ${ }^{3.4}$. This is not because of 'failure' of the Glu protomers to polymerize, but rather to the virtual absence of Glu protomers in vivo (we have determined $^{4}$ that $<2$ per cent of the protomeric pool of tubulin is detyrosinated). This probably results from the rapid retyrosination of Glu tubulin in the monomer pool, as microinjected Glu protomer or Glu protomer resulting from the depolymerization of existing Glu microtubules is rapidly retyrosinated ${ }^{2.4}$.

How, then, do microtubules with different levels of Glu tubulin arise in a common cytoplasm? Our experiments, originally reported two years $\mathrm{ago}^{3}$ and elaborated on in a detailed report ${ }^{4}$, demonstrate that microtubules distinct in their content of Glu tubulin are generated from a cycle (see figure) that involves polymerization of $\mathrm{Tyr}$ protomers, postpolymerization detryosination of $\mathrm{Tyr}$ microtubules, breakdown of the resulting Glu-enriched microtubules and efficient retyrosination of Glu protomers.

In his article, Burns predicted that cellular microtubules would contain "a steadily increasing proportion of Glu $\alpha / \beta$ dimers with time, and hence with distance along the microtubule". It is obvious from the cycle we have proposed that growing miviotubules would exhibit a gradient of Glu/Tyr ratios along their lengths. The slope and relative position of this gradient along the microtubule would depend upon the rate of polymerization of Tyr protomers and the rate of detyrosination by tubulin carboxypeptidase. However, we would not expect such a gradient to occur on microtubules that have amassed high levels of Glu tubulin, as these microtubules are not growing in vivo ${ }^{5}$ and, in fact, persist without addition of Tyr protomers for more than 16 hours $^{6}$. This extraordinary longevity of Gluenriched microtubules contrasts with that of most cellular microtubules, which persist less than 10 minutes. Whether Glu tubulin level is a cause or merely an effect of microtubule stability, as Burns points out, is unknown.

In summary, existing data answer the question Burns posed concerning the mechanism of tyrosination/detyrosination. Further work should elucidate these unusual post-translational modifications: the most likely possibilities include the idea that Glu tubulin, which predominates in 'old' microtubules, is recognized by one or more specific microtubule-associated proteins to allow specialized function or interaction of that microtubule.

Jeannette Chlöe Bulinski GregG G. Gundersen

Department of Biology,

University of California,

Los Angeles, California 90024, USA

DANIEL R. WEBSTER

Laboratory of Molecular Biology,

University of Wisconsin,

Madison, Wisconsin 53706, USA

1. Burns, R. Nature 327: 103-104 (1987).

2. Webster, D.R., Gundersen, G.G., Bulinski, J.C. \& Borisy, G.G. J. Cell Biol. 101, 150a (1985)

3. Gundersen, G.G., Khawaja, S. \& Bulinski, J.C. J. Cell Biol. 101, 257a (1985).

4. Gundersen, G.G., Khawaja, S. \& Bulinski, J.C. J. Cell Biol (in the press)

5. Gundersen, G.G., Khawaja, S. \& Bulinski, J.C. in The Cytoskeleton and Cell Differentiation and Development (eds Maccioni, R.B. \& Arechaga, J.) (ICSU, Miami, 1987).

6. Webster, D.R., Gundersen, G.G., Bulinski, J.C. \& Borisy, G.G. J. Cell Biot. 103, 270a (1986).

\section{RNA world}

SIR-Prompted by the following quotes, I have written a short verse that may appeal to Nature readers.

"It was long thought that every cellular reaction is catalysed by a protein enzyme. The discovery that RNA can cut, splice and assemble itself overturns the principle - and throws light on early evolution."

$$
\text { Scientific American } 255 \text { (5), } 64 \text { (1986). }
$$

"[Cech's] discovery that RNA molecules can ... act as enzymes makes the notion that life began from RNA very attractive."

E.G. Nisbet

Nature 322, 206 (1986)

Primaeval proteins, earliest of enzymes,

Step aside. On the hydrothermal plane

It's an RNA world.

Slithering through the Archaean soup,

Improbable chains dabbed on ancient clays

Stain the basalt fabric,

And grimly splice themselves to bits.

Naturally selected, each is a list,

A preordained script;

Crudely but with dignity

Replicating catalytically. Stranded,

The detached daughters cling to rock.

Against the open ocean current, a coat of lipids,

Thank you, would be welcome shelter.

Bruce Ganem

Cornell University,

Department of Chemistry,

Baker Laboratory,

Ithaca, New York 14853, USA 